\title{
Improving paediatric prescribing practice in a district general hospital through implementation of a quality improvement programme
}

\author{
Peter Donnelly, Sara Lawson, Claire Watterson \\ Altnagelvin Area Hospital. Western Health \& Social Care Trust. Northern Ireland
}

\begin{abstract}
Prescribing errors are a well recognised cause of adverse incidents and have a direct effect on patients.[1] This impacts on the doctor-family relationship and results in breakdown of trust and communication.[2] This quality improvement project was carried out in the paediatric ward of a district general hospital in Northern Ireland. A retrospective analysis of paediatric prescribing errors between January and December 2013 identified two errors that were felt to be secondary to under-reporting. A baseline audit was subsequently performed that highlighted 32 errors across 12 drug charts.
\end{abstract}

A driver diagram identified three components contributing to prescribing errors and relevant tests of change were developed. The three primary drivers included: education and communication, practical prescribing changes, and medicine reconciliation. Seven interventions were implemented sequentially over a six month period and their effectiveness assessed by a prospective drug chart audit. Ten drug charts were selected at random by the staff nurse allocated to medications on the day of audit. The charts were audited using a predesigned proforma and the total number of errors counted. These were subcategorised and results displayed in graphical format after each intervention.

Seven audit cycles were completed in total after each intervention was put into practice. The number of errors (including percentage change following each intervention) is as follows: intervention 1: 32 (+19\%); Intervention 2: 31 (+15\%); Intervention 3: 17 (-37\%); Intervention 4: 12 (-56\%); Intervention 5: $15(-44 \%)$; Intervention 6: 7 (-74\%); Intervention 7: 10 (-63\%). In conclusion, permanent and successful measures are needed to reduce prescribing errors in order to minimise the impact of staff changeover and knowledge deficits.

\section{Problem}

Anecdotal evidence suggested a number of prescribing errors were occurring within the paediatric ward of a district general hospital resulting in patient and medication safety concerns. The existing incident reporting system was reviewed and felt to underestimate medication prescribing errors as evidenced by a review of submitted incident reports. A retrospective analysis of paediatric prescribing errors between January and December 2013 identified two errors, felt to be secondary to under reporting.

A baseline audit was subsequently performed that reviewed patient demographics, allergy status, generic prescribing, drug dosage, timings, drug reconciliation, antibiotic prescribing (indication and duration), and legibility. Twelve drug charts were analysed highlighting 32 errors - a significant increase from the amount of errors officially reported. The clinical impact of these errors was variable however there was significant potential to cause a serious adverse incident affecting both the patient and the healthcare team. This problem was confounded by the absence of a ward pharmacist who has been appointed but had yet to start work.

\section{Background}

Previous research has acknowledged that paediatric patients are at risk of encountering medication errors including prescribing errors.[3] More recent research regarding paediatric prescribing has targeted the use of electronic prescribing in paediatric practice.[4] In reality, not all institutions have the resources necessary to implement electronic prescribing and it is for this reason that quality improvement projects are required to look broadly at how prescribing practice could be improved. A recent systematic review on this subject concluded that it is difficult to determine what constitutes an optimal intervention and recommends more standard studies in the future.[5]

There is existing evidence that providing targeted and multidisciplinary feedback to clinicians and students results in improved prescribing practice.[6,7] It is important to realise that doctors rotate to different hospital sites throughout training and therefore it is important to address process as well as outcome measures to create a sustained improvement. This project aims to provide clinician education as well as create interventions that will help to improve prescribing practice.

\section{Baseline measurement}

A retrospective analysis of paediatric prescribing errors from incident report forms submitted between January and December 2013 identified two errors, both felt to be secondary to under reporting. Subsequently a baseline audit was performed reviewing patient demographics, allergy status, generic prescribing, drug dosage, timings, drug reconciliation, antibiotic prescribing (indication and duration), and legibility. Twelve drug charts were analysed using the designed proforma and 32 errors were noted. 
Twelve drug charts were initially analysed as this represented the number of paediatric inpatients on the ward for that day. Future audit cycles reviewed 10 drug charts per cycle and so to make our results comparable we averaged the 32 errors across 12 drug charts to equate to 27 errors within 10 .

See supplementary file: ds5500.png - "Picture 1: A table showing the results of a baseline kardex audit"

\section{Design}

A driver diagram identified three components contributing to prescribing errors and relevant tests of change were developed. The three primary drivers included education and communication, practical prescribing changes, and medicine reconciliation.

This project was discussed with the cooperation and working together group as part of a cross-border patient safety group. As a result of these discussions a quality improvement project was performed using plan, do, study, act (PDSA) cycles and multiple audit cycles.

In total the project spanned six months with seven tests of change being implemented over a three month period. These comprised of: presentation of baseline data, staff completion of an online learning module, introduction of a safety notice board, inclusion of data to the nursing safety brief, introducing a medication administration checklist, ensuring staff access to a regional patient information system, appointment of a ward pharmacist, and finally developing a pocket sized antibiotic reference tool.

Each intervention was implemented sequentially and effectiveness assessed by a prospective drug chart audit. Ten drug charts were selected at random by the staff nurse allocated to medications on the day of audit. Results were collated in a run diagram in order to disseminate progress to the team. It is important to note that some of the interventions were one-offs and some were continuous and the measured effect would be cumulative.

\section{Strategy}

Seven interventions were implemented in the form of PDSA cycles. Each of these small tests of change were reviewed by subsequent audit against the proforma used for our baseline audit. Each audit was performed at least seven days after a new change was implemented in order to let the intervention take effect. It was decided that ten charts would be reviewed on each occasion and the results collated in a run diagram.

Intervention 1 was the presentation of the results of our baseline audit. The aim was to begin developing a culture of awareness and education. We also aimed to highlight the significant scale of the problem.

Intervention 2 was the completion of an online learning module regarding prescribing. This was a free online module and so access to the learning was easily accessible. Each member of medical staff was asked to complete the module and provide a certificate of completion. Over $70 \%$ of medical staff completed this module.

Intervention 3 was the introduction of a safety notice board. This board was used to highlight the results of the most recent PDSA cycle. We also updated a run diagram on this notice board so that the team were aware of the progress we were making. The various types of errors that were being made were also listed on this board. Members of staff were not individually named in terms of the errors they made. This was felt to be detrimental to the creation of a learning environment.

Intervention 4 was two fold. Our data were included into a separate nursing safety brief to ensure all members of the healthcare team were aware of the team performance. A checklist for medication safety was also added to the medication trolley at this time to help raise awareness during the medicines administration process.

Intervention 5 was ensuring that medical staff had access to an electronic care record (ECR) which is a system that enables clinicians to review prescriptions from patients' general practitioner. This was felt to help address concerns regarding medicines reconciliation.

Intervention six was the introduction of a ward pharmacist. As mentioned earlier, funding had been received for a ward pharmacist outside of the remit of this project. The introduction of a pharmacist was felt to potentially have a significant impact upon our audit results and so was considered as an intervention for the purposes of this study.

The final intervention was the introduction of a pocket sized antibiotic guideline for prescribing staff. This acted as a reminder for clinicians to consider the choice and duration of each antibiotic prescribed.

\section{Results}

The various changes led to a global improvement in paediatric prescribing; a reduction in errors of $63 \%$.

When we equated our baseline audit to 27 errors across ten charts, we noted an increase to 32 errors after our first intervention. This represents an increase in errors of $19 \%$. There was slight improvement after our second intervention (completion of an online learning module) with 31 errors identified. However, this still represents an increase in errors from our baseline data of $15 \%$. Introduction of a safety notice board resulted in a significant improvement in performance with 17 errors noted which equate to an improvement of $37 \%$ from our baseline. This global improvement continued with our fourth intervention, introduction of a medication administration checklist, with an improvement of $56 \%$ from baseline (12 errors). The fifth intervention was not successful in itself however due to a cumulative effect of other interventions a global reduction of $44 \%$ was still achieved. The appointment of a ward pharmacist (the funding of which was outside the remit of this project) resulted in ongoing reduction of errors $(74 \%$, or seven errors). The final intervention, a pocket sized antibiotic reference 
tool, was again not individually successful but a sustained improvement was achieved from baseline with 10 errors recorded across 10 charts; an improvement of $63 \%$ from the initial audit.

This represents a significant improvement in prescribing practice, thereby reducing the potential impact on patients of prescribing errors. The main difficulty encountered during the PDSA cycles was regarding resistance to change and buy-in from junior medical staff. Continued encouragement helped to address these issues.

See supplementary file: ds5501.png - "A run diagram showing the impact of each intervention on prescribing practice."

\section{Lessons and limitations}

A number of lessons were learnt through the duration of this project.

We noted that there were unsuccessful PDSA cycles: presentation of baseline data, completion of an online learning module, staff access to ECR, and the introduction of handheld antibiotic guidelines. The remainder of the PDSA cycles appeared to be globally effective. These interventions targeted various areas within our initial driver diagram so it is difficult to ascertain the reasons why they were unsuccessful. It is felt by the team that various factors such as time pressure may have played a role and this reinforces the difficulty in finding optimal interventions. The biggest challenge faced during this project was surrounding buy-in from medical and nursing staff. Senior staff and those with an interest in patient safety were proactive in encouraging more junior team members.

A limitation is that his quality improvement project was performed in the six months prior to changeover of staff. This project showed that education can improve prescribing practice however further work is required to ensure any changes are sustainable. Some of the PDSA cycles involved continuous changes whereas some interventions were one-offs. Further ongoing work is needed to help address sustainability within the trust. A factor to be considered when reviewing the results is that each intervention would have a cumulative effect from any previously implemented interventions.

Each of the planned interventions were cost effective as no external funding was required and the interventions did not involve excessive time pressures to staff. However, it must be factored in that external funding had already been approved for the appointment of a pharmacist outside of our planned interventions. The time taken to complete the online learning module was felt by those involved to be minimal as the module was completed within thirty minutes by each staff member involved.

\section{Conclusion}

Significant improvements were made when we targeted the primary driver of communication and education. We believe that a focus of education and awareness will help create an ethos of patient safety and a climate for change within the trust.
We need to introduce permanent and successful measures to reduce prescribing errors in order to minimise the impact of staff changeover and knowledge deficits. The most significant change followed the introduction of a patient safety notice board which reduced the number of errors from 31 to 17 . This represents a percentage reduction of $45 \%$ following a single intervention. It follows from this that education and awareness is paramount to continued professional development and quality improvement.

\section{References}

1. Neale G, Woloshynowych M, Vincent $C$. Exploring the causes of adverse incidents in NHS hospital practice. J R Soc Med July 2001; (94):322-30.

2. Mazor KM, Reed GW, Yood RA et al. Disclosure of Medical Errors. What Factors Influence How Patients Respond. J Gen Intern Med May 3006; (21): 704-10.

3. Miller MR, Robinson KA, Lubomski LH, Rinke ML, Pronovost $\mathrm{PJ}$. Medication errors in paediatric care: a systematic review of epidemiology and an evaluation of evidence supporting reduction strategy recommendations. Qual Saf Health Care 2007 April; 16(2): 116-26.

4. Johnson KB, Lehmann CU, The Council on Clinical Information Technology et al. Electronic Prescribing in Pediatrics: Toward Safer and More Effective Medication Management. Pediatr 2013 April; 131(4): e1350-6.

5. Rinke ML et al. Interventions to reduce pedicatric medication errors: a systematic review. Pediatr 2014 Aug;134(2):338-60.

6. Eisenhut M, Sun B, Skinner S. Reducing Prescribing Errors in Paediatric Patients by Assessment and Feedback Targeted at Prescribers. Pediatr 2011; 2011: 545681.

7. Stewart M, PurdyJ, Kennedy N, Burns A. An interprofessional approach to improving paediatric medication safety. BMC Medic Educ 2010; 10:19.

\section{Declaration of interests}

Nothing to declare.

\section{Acknowledgements}

Mary Ledwidge, cooperation and working together team, medical and nursing staff of ward 6, Altnagelvin Area Hospital.

\section{Ethical approval}

Ethical approval was not sought for this project. This work was deemed to be an improvement study and not a study on human subjects and therefore ethical approval was not required - as per local policy. 